\title{
Sullo sfondo della Costituzione. Dalle norme prescritte alle norme presupposte
}

Angela Ferrari e Filippo Pecorari

PUBBLICATO: 17 AGOSTO 2021

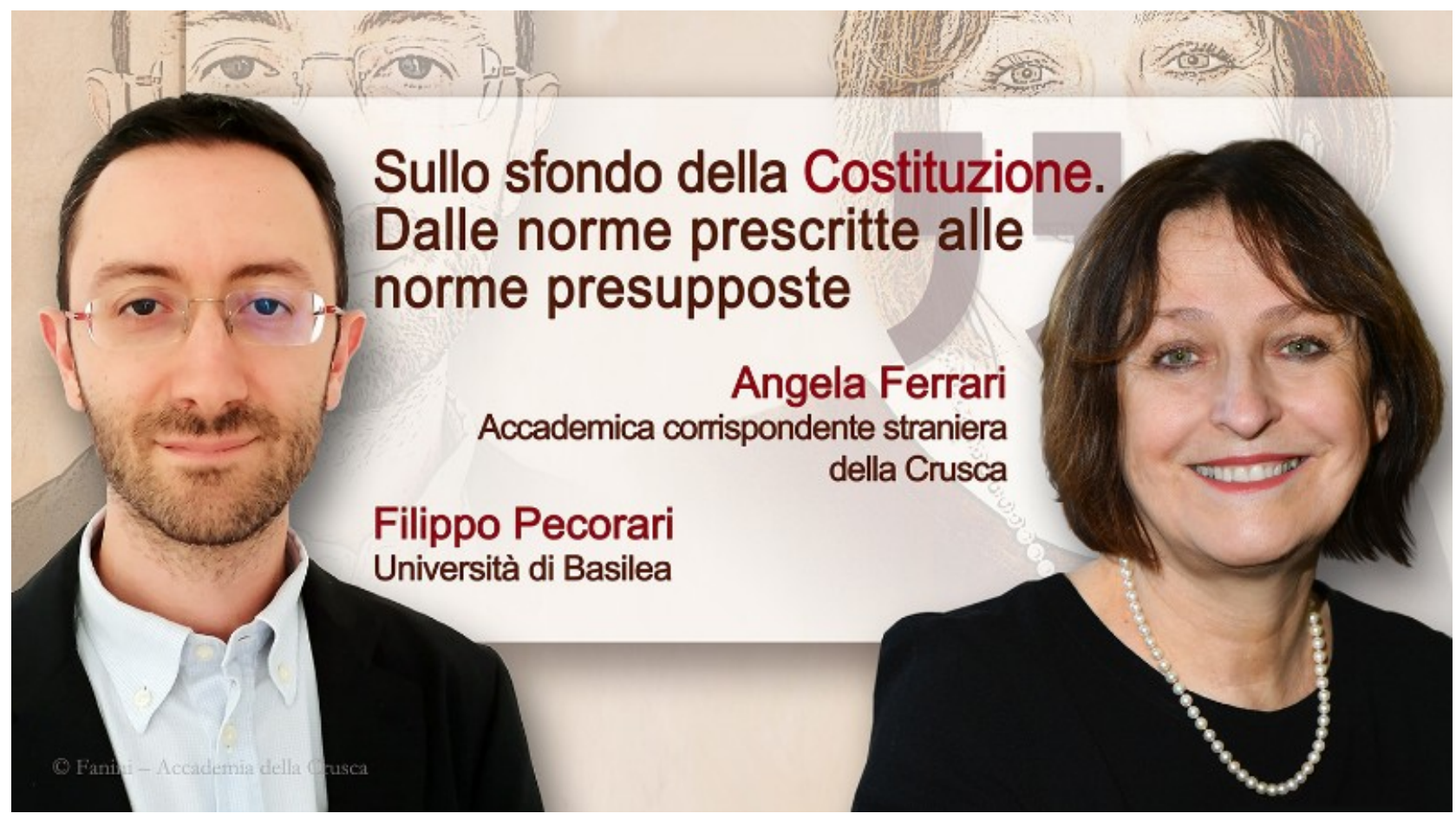

$\mathrm{P}$

rendiamo la prima parte dell'articolo 2 della Costituzione italiana: La Repubblica riconosce e garantisce i diritti inviolabili dell'uomo [...]. Questo articolo non ci dice solo quello che afferma esplicitamente, e cioè che lo Stato riconosce e garantisce i diritti inviolabili dell'uomo; ci comunica anche un'altra cosa: che la Repubblica considera che ci siano, che esistano diritti dell'uomo che vanno considerati come inviolabili. Si tratta di un'asserzione implicita, che sta sullo sfondo rispetto a quella esplicita, ma che non per questo è più debole, anzi. Prima di tutto se non fosse vera, l'asserzione esplicita non avrebbe senso: come potrebbe, la Repubblica, riconoscere e garantire qualcosa che non esiste? In secondo luogo, quest'asserzione implicita rimane vera anche se noi neghiamo o interroghiamo l'enunciato principale. Se qualcuno ci chiedesse Ė vero che la Repubblica riconosce e garantisce $i$ diritti inviolabili dell'uomo?, non metterebbe assolutamente in dubbio che questi diritti esistono.

A questi contenuti che stanno sullo sfondo dell'enunciazione, che sono necessari perché l'enunciazione abbia un senso e che sopravvivono alla negazione o allinterrogazione, le scienze linguistiche danno il nome di "presupposizioni": sono un tipo particolare di significato implicito, che si aggiunge ai molti altri significati impliciti che accompagnano il nostro parlare e il nostro scrivere quotidiani o estemporanei. Dal punto di vista linguistico, la peculiarità di questo tipo di implicito sta nell'essere legato a particolari costruzioni. La presupposizione esistenziale vista sopra è per esempio connessa con l'articolo determinativo; un'espressione verbale come Ho smesso di fumare presuppone che, prima, fumassi; un costrutto come É Giorgio che ha rotto il vetro presuppone che qualcuno abbia rotto il vetro e asserisce che quel qualcuno è Giorgio. 
Le presupposizioni sono preziose dal punto di vista comunicativo. Permettono per esempio di mantenere compatta l'informazione: se la lingua non potesse contare su di esse, si dovrebbe esplicitare tutto, e l'articolo 2 della Costituzione suonerebbe cosi: L'uomo ha diritti inviolabili e la Repubblica li riconosce e li garantisce; l'enunciato Ho smesso di fumare diventerebbe, con una ridondanza improbabile, Prima fumavo e adesso ho smesso. O ancora, le presupposizioni permettono di far passare un'informazione tra le righe, di dire senza dire veramente: se affermo Non so se questa costruzione eterogenea piacerà a Giorgio, affermo anche che per me la costruzione è eterogenea, ma non lo faccio in modo diretto, lo dico en passant.

Come tutti i testi, anche la nostra Costituzione è disseminata di presupposizioni. Data la sua trasparenza e la sua chiarezza esemplari, questo fatto potrebbe suonare strano, eppure è così: la Costituzione presuppone contenuti mettendoli in vario modo al servizio dei suoi obiettivi comunicativi e giuridici. Ma quali sono le forme e le funzioni principali delle presupposizioni nella legge fondamentale dello Stato italiano?

La maggior parte delle presupposizioni presenti nella Costituzione è generata da espressioni nominali con l'articolo determinativo, che svolgono almeno tre funzioni significative. Una prima funzione notevole è quella di introdurre principi ideali, di natura etica, politica o sociale, che la presupposizione presenta come valori di base della democrazia: valori talmente basilari da non avere bisogno di essere asseriti esplicitamente nella Costituzione. È il caso dei diritti inviolabili delluomo menzionati nell'articolo 2, ma anche di molte altre espressioni. Per fare qualche esempio: $i$ doveri inderogabili di solidarietá politica, economica e sociale (sempre nell'art. 2), la libertá personale (art. I3), il senso di umanità (art. 27), l'utilitá sociale (art. 4I). Un caso particolarmente interessante è quello dell'articolo 32, in cui si dice che la legge non puó in nessun caso violare i limiti imposti dal rispetto della persona umana: questa formulazione presuppone, al tempo stesso, che esiste un valore fondamentale come il rispetto della persona umana, e che questo principio etico impone dei limiti alla legge.

Con l'articolo determinativo sono introdotti nella Costituzione non solo valori di base della democrazia, ma anche - ed è la seconda funzione del costrutto - altri concetti rilevanti dal punto di vista giuridico. Si pensi per esempio, per quanto riguarda l'ambito del lavoro, all'articolo 37: [...] La Repubblica tutela il lavoro dei minori con speciali norme [...]. L'articolo presuppone, con la stessa modalità dei casi precedenti, l'esistenza del lavoro dei minori. L'aspetto più significativo di un tale esempio è che, attraverso la presupposizione, il testo non si limita a comunicare implicitamente l'esistenza del lavoro dei minori: esso comunica anche la sua legittimità. Non bisogna dimenticare, infatti, che la Costituzione è un testo normativo: la sua funzione è stabilire una serie di norme su cui si basa la vita associata in una comunità. Tutti gli oggetti, le entità e i concetti menzionati in un testo con queste caratteristiche sono per definizione oggetti, entità e concetti ammessi dalla legge. L'articolo 37 presuppone dunque, senza dirlo in maniera esplicita, che in Italia il lavoro dei minori è legittimo. In questo modo, unopzione che di per sé sarebbe discutibile sul piano etico-sociale viene presentata come un dato di fatto, e cosi schermata da ogni discussione. C'è solo un'eccezione alla validità di questa classe di presupposizioni: il caso in cui la legittimità è vietata esplicitamente dall'enunciato, come accade ad esempio nell'articolo 27 (Non é ammessa la pena di morte).

La presupposizione legata all'articolo determinativo si manifesta anche in una terza classe di espressioni, molto diversa dalle precedenti. Sono numerosi gli articoli della Costituzione che rimandano all'esterno della Carta, presupponendo l'esistenza di altre norme che in vario modo dovranno completare o precisare il testo costituzionale. È per esempio il caso dell'articolo 5r: Tutti $i$ cittadini dell'uno o dell'altro sesso possono accedere agli uffici pubblici e alle cariche elettive in condizioni di eguaglianza, secondo i requisiti stabiliti dalla legge [...]. Il cuore dell'articolo è la prescrizione che tutti i 
cittadini, indipendentemente dal sesso, debbano avere le stesse possibilità di accesso agli uffici pubblici e alle cariche elettive. L'articolo comunica però anche qualcosa in più: il rispetto della norma è vincolato a specifici requisiti, che - si presuppone - saranno stabiliti in un'altra legge. Esempi come questo mostrano chiaramente il ruolo fondativo della Costituzione, anche in termini di priorità temporale sulle altre leggi: la legge fondamentale dello Stato può presupporre l'esistenza di norme che in realtà non esistevano ancora al momento della stesura del testo (e in parte non esistono ancora neppure oggi), ma che in un momento successivo dovranno entrare a far parte dell'ordinamento legislativo della Repubblica.

A conclusione di questa breve rassegna di significati impliciti, menzioniamo un ultimo esempio che ci porta ad allargare lo sguardo a un diverso tipo di presupposizione. L'articolo 5 della Costituzione recita: La Repubblica, una e indivisibile, riconosce e promuove le autonomie locali [...]. Che cosa comunica esattamente l'articolo? Senz'altro che la Repubblica riconosce e promuove le autonomie locali; ma anche, in maniera meno diretta, che la Repubblica è una e indivisibile. Quest'ultimo significato è trasmesso attraverso una coppia di aggettivi racchiusi tra virgole, a margine dell'asserzione esplicita centrale. Anche un contenuto come questo può dirsi presupposto: se si prova a negare l'enunciato nel suo complesso (ad esempio dicendo Non è vero che la Repubblica, una e indivisibile, riconosce e promuove le autonomie locali), il fatto che la Repubblica sia una e indivisibile rimane vero. Può forse sembrare che questo fenomeno costituisca una raffinatezza linguistica poco significativa sul piano giuridico, ma non è cosi. Prova ne è il fatto che, nei lavori dell'Assemblea Costituente, la questione fu al centro di un acceso dibattito: mentre alcuni proponevano di dare maggiore rilievo all'indivisibilità della Repubblica modificando l'articolo i della Carta (e adottando la formulazione "L'Italia è una Repubblica democratica e indivisibile"), altri - tra cui il Presidente della Commissione per la Costituzione Meuccio Ruini - spinsero per l'adozione della formulazione attualmente vigente. E il loro argomento vincente fu proprio una riflessione sul valore comunicativo dei contenuti espliciti e impliciti: la proposta di modifica dell'articolo I, asserendo esplicitamente l'indivisibilità della Repubblica, avrebbe potuto far pensare che tale principio fosse in pericolo; meglio presentarlo come presupposto, e darne cosi per scontata la validità.

\section{Cita come:}

Angela Ferrari e Filippo Pecorari, Sullo sfondo della Costituzione. Dalle norme prescritte alle norme presupposte , "Italiano digitale", XVIII, 2021/3 (luglio-settembre)

DOI: $10.35948 / 2532-9006 / 2021.10597$

Copyright 2021 Accademia della Crusca

Pubblicato con licenza creative commons CC BY-NC-ND 